\title{
Prediction of DNA binding proteins using local features and long-term dependencies with primary sequences based on deep learning
}

\author{
Guobin Li ${ }^{1}$, Xiuquan Du ${ }^{\text {Corresp., } 2}$, Xinlu Li ${ }^{1}$, Le Zou ${ }^{1}$, Guanhong Zhang $^{1}$, Zhize Wu $^{1}$ \\ ${ }^{1}$ School of Artificial Intelligence and Big Data, Hefei University, Hefei, China \\ 2 School of Computer Science and Technology, Anhui University, Hefei, China \\ Corresponding Author: Xiuquan Du \\ Email address: dxqllp@163.com
}

DNA-binding proteins (DBPs) play pivotal roles in many biological functions such as alternative splicing, RNA editing, and methylation. Many traditional machine learning (ML) methods and deep learning (DL) methods have been proposed to predict DBPs. However, these methods either rely on manual feature extraction or fail to capture long-term dependencies in the DNA sequence. In this paper, we propose a method, called PDBPFusion, to identify DBPs based on the fusion of local features and long-term dependencies only from primary sequences. We utilize convolutional neural network (CNN) to learn local features and use bi-directional long-short term memory network (Bi-LSTM) to capture critical long-term dependencies in context. Besides, we perform feature extraction, model training, and model prediction simultaneously. The PDBP-Fusion approach can predict DBPs with $86.45 \%$ sensitivity, $79.13 \%$ specificity, $82.81 \%$ accuracy, and 0.661 MCC on the PDB14189 benchmark dataset. The MCC of our proposed methods has been increased by at least $9.1 \%$ compared to other advanced prediction models. Moreover, the PDBP-Fusion also gets superior performance and model robustness on the PDB2272 independent dataset. It demonstrates that the PDBP-Fusion can be used to predict DBPs from sequences accurately and effectively; the online server is at http://119.45.144.26:8080/PDBP-Fusion/. 
2 Prediction of DNA binding proteins using local features

3 and long-term dependencies with primary sequences

4 based on deep learning

5

6

\author{
Guobin $\mathrm{Li}^{1}$, Xiuquan $\mathrm{Du}^{2}$, Xinlu $\mathrm{Li}^{1}$, Le Zou ${ }^{1}$, Guanhong Zhang ${ }^{1}$ and Zhize $\mathrm{Wu}{ }^{1}$ \\ ${ }^{1}$ School of Artificial Intelligence and Big Data, Hefei University, Hefei 230601, China \\ ${ }^{2}$ School of Computer Science and Technology, Anhui University, Hefei 230601, China
}

Corresponding Author:

Xiuquan $\mathrm{Du}^{2}$

Anhui University, Hefei 230601, China

Email address: dxq1lp@163.com

\title{
Abstract
}

DNA-binding proteins (DBPs) play pivotal roles in many biological functions such as alternative splicing, RNA editing, and methylation. Many traditional machine learning (ML) methods and deep learning (DL) methods have been proposed to predict DBPs. However, these methods either rely on manual feature extraction or fail to capture long-term dependencies in the DNA sequence. In this paper, we propose a method, called PDBP-Fusion, to identify DBPs based on the fusion of local features and long-term dependencies only from primary sequences. We utilize convolutional neural network (CNN) to learn local features and use Bi-directional long-short term memory network (Bi-LSTM) to capture critical long-term dependencies in context. Besides, we perform feature extraction, model training, and model prediction simultaneously. The PDBP-Fusion approach can predict DBPs with $86.45 \%$ sensitivity, $79.13 \%$ specificity, $82.81 \%$ accuracy, and $0.661 \mathrm{MCC}$ on the PDB14189 benchmark dataset. The MCC values of our proposed methods had been increased by at least $9.1 \%$ compared to other advanced prediction models. Moreover, the PDBP-Fusion also gets superior performance and model robustness on the PDB2272 independent dataset. It demonstrates that the PDBP-Fusion can be used to predict DBPs from sequences accurately and effectively; the online server is at Http:// 119.45.144.26:8080/PDBP-Fusion/.

\section{Introduction}

Protein-DNA interactions are widespread in all living organisms. A variety of biological processes are involved in these interactions including DNA replication, DNA repair, viral infection, DNA packaging, and DNA modification [1]-[3]. In recent years, a larger number of DBP sequences have been generated by rapid advances in genomic and proteomic techniques. Exploring how protein-DNA interactions occur help us understand the genome.

In the early days, many experimental techniques have been proposed to predict DBPs. However, due to the time-consuming and money-consuming disadvantages of the experimental method, computational 
40

41

42

43

44

45

46

47

48

49

50

51

52

53

54

55

56

57

58

59

60

61

62

63

64

65

66

67

68

69

70

71

72

73

74

75

76

77

78

79

80

81

82

83

84

methods show great advantages in processing sequence data. So far, many effective computational prediction methods have been developed to identify DNA-binding proteins. They mainly use classic machine learning prediction models combined with complex feature extraction techniques. These methods include support vector machines (SVMs) [4]-[11], artificial neural networks (ANN) [12], [13], decision tree [14], Bayesian network [15], and Random forest (RF) [16]-[19]. Support vector machines (SVM) and Random forest (RF) algorithm are widely used as classifiers for predicting DBPs and get better performance. Many sequence-based methods and web servers have been developed to identify DBPs. Recent methods and server names among them are: PseDNA-Pro [20], Local-DPP [21], SVM-PSSM-DT [22], BindUP [23], PSFM-DBT [24], HMMBinder [25], iDNAProt-ES [26], DBPPred-PDSD [27], MSFBinder [28], DP-BINDER [29], and HMMPred [30].

In recent years, stack generalization (stack) as an integrated learning technique has gained much attention from researchers. StackDPPred [20] first used features extracted from PSSM and residue-specific contact energy and then trained a stack-based machine learning method to predict DBPs. PredDBP-Stack [21] improved DBP prediction performance by exploring valuable features from the HMM profile. StackPDB [22] took fusion features such as EDT, RPT, PseAAC, PsePSSM, and PSSM-TPC and then applied the stacked ensemble classifier to predict DBPs.

More and more evidence shows that it is practical to predict DBPs only from primary sequences. The traditional machine learning method shows superiority in solving the problem of small-scale data classification [5], [6]. Unfortunately, these methods required the use of well-designed sequence features and evolutionary information features. They also need to be supported by relevant professional knowledge and experience. Additionally, feature extraction, training and forecasting cannot be performed simultaneously.

Recently, deep learning has been successfully applied to many big dataset classification tasks [23][26]. Deep learning technology has incomparable advantages in the computation of large-scale DNA sequence data. For examples, Alipanahi and Delong first proposed DeepBind model based on deep learning technology to predict DNA binding proteins [27]. Zeng et al. predicted DNA binding sites based on CNN and many transcription factor data. They determined the best performance network structure by changing the CNN network width, depth, and pool operation design [28]. Zhou et al. developed the CNNSite model based on a neural network and combined it with captured sequence features and evolution features to predict sequence binding residues [29]. Shadab et al. proposed DeepDBP-ANN and DeepDBP-CNN models to identify DBPs. The former used a generated feature set trained on the traditional neural network. The latter used a previously learned embedding and a convolution neural network [30].

Although several CNN based methods have been developed to predict DBPs, they are not good enough to achieve better accuracy in DBPs prediction. For instance, Zhang et al. developed the DeepSite model, which used Bi-LSTM and then CNN to capture long-term dependencies between the sequence motifs in DNA [31]. Qu et al. used word embedding to encode sequences and then used CNN and LSTM as a classifier to predict DBPs [32]. Hu et al. proposed CNN-BiLSTM method to identify DBPs. They coordinated a Bi-LSTM and a CNN [33] and also used word embedding technology. Recently, Du et al. developed a deep learning method named MsDBP [34], which obtained four-vectors (multi-scale features) based on $25 \%, 50 \%, 75 \%$, and $100 \%$ of the original sequence length. Moreover, they used many dense layers to learn different abstract features for predicting DBPs [34].

These methods based on CNN can only represent the local dependence of the DNA sequence information, but the long-term dependence of the sequence is not considered. It is a better choice to predict DBPs by fusing the local features obtained by $\mathrm{CNN}$ with the long-term context-dependent features captured by Bi-LSTM. Although some existing methods also combined CNN and LSTM to predict DBPs, they both

Peer] reviewing PDF | (2020:08:52191:1:1:NEW 3 Mar 2021) 
85

86

87

88

89

90

91

92

93

94

95

96

97

98

99

100

101

102

103

104

105

106

107

108

109

110

111

112

113

114

115

116

117

118

119

120

121

122

completed the preprocessing of gene sequences based on word embedding. The One-hot encoding method used in this paper is simple and effective, and the pre-processing is more efficient. In addition, we also need to consider the design of a convolutional neural network with the size, characteristics, and distribution of the sequence data.

In this study, we develop the PDBP-Fusion method to overcome the disadvantages of the existing methods. One-hot encoding was used in this approach, which is easier and faster than word embedding. Moreover, CNN was used to obtain the local features of DNA sequences through self-learning, and BiLSTM was used to capture long-term dependencies in the sequence context. Finally, a fusion feature combining local and global characteristics was used to predict DBPs. The contributions of this research are described as follows:

(1) Since the length of the DNA sequence variation, the optimal sequence length is determined using experiments to obtain the best sequence characteristics. In this article, a grid search method based on the sequence length distribution is suggested to search for the best truncated sequence length parameters.

(2) A new method, PDBP-Fusion, has been developed based on deep learning to predict DBPs. It consists of a CNN network and a Bi-LSTM network. The former is responsible for extracting the abstract features layer by layer. Simultaneously, the latter is responsible for obtaining long-term dependencies in the sequence context.

(3) The proposed method does not require manual extraction of data features; it uses only deep learning to self-learn original sequence features based on the primary sequence. Two coding practices, One-hot and word embedding, were used to predict DNA binding sites. The optimal network structure was found by a parameter grid-search strategy based on the benchmark data set for predicting DBPs.

\section{Materials \& Methods}

In this section, we first introduce the benchmark dataset and the independent test dataset. Next, we describe our proposed PDBP-Fusion framework. Finally, we illustrate all the details concerning the construction of the model, the evaluation of the model and the experimental setting of the parameters.

\subsection{Datasets}

We use the benchmark dataset obtained from Ma et al. [18] referred to as PDB14189. The PDB14189 dataset is composed of 7129 DBPs (positive samples) and 7060 non-DBPs (negative samples). All of them are from the UniProt [46] database. This dataset is identical to MsDBPs [45].

In addition, we used an independent test dataset, PDB2272, to compare the performance of our proposed model with other existing prediction methods [19][35][36][34]. We obtained original dataset consisting of 1153 DBPs and 1153 non-DBPs from Swiss-Prot. We removed sequences that had more than $25 \%$ similarity and filtered out sequences with irregular characters ("X" or "Z"). Finally, the PDB2272 dataset contained 1153 DBPs and 1119 non-DBPs.

\subsection{Framework of the PDBP-Fusion model}

In this study, we develop a deep learning model called PDBP-Fusion, which combined CNN and BiLSTM, to predict DNA binding proteins. The former obtained local DNA sequences through self-learning and the latter learned the long-term dependencies in the sequence context. The proposed models consist of 
123

the sequence encoding layer (one-hot encoding or embedding encoding), the local feature learning layer, the long-term context learning layer, and the synthetic prediction layer. Figure 1 illustrates the main framework of the PDBP-Fusion model.

\subsubsection{Sequence encoding}

Feature coding is a critical task in building machine learning models. And it is preferable to obtain a suitable coding scheme after observing the characteristics of the dataset. The statistical results showed that the sequence length of the DNA benchmark database ranged from 50 to 2743 . The sequence length distribution is provided in Figure 2.

Since the sample length of the DNA sequence varies, it is necessary to choose an appropriate maximum sequence length for data processing. A maximum length was chosen as the best experimental endpoint reference for the PDBP-Fusion model, as determined by a series of experiments between 100 and 1000 . Further details on the results of the comparison experiment are available in section 3.2.

(a) One-hot encoding

One-hot encoding is a general method that can vectorize any categorical features. In the One-hot encoding method, amino acid must be encoded numerically.

For example, a DNA sequence "S= EFDYVICEEE" was taken from Fig. 3(a) and encoded with the One-hot approach. An output vector, with a dimension of $21 \%$ d, was obtained from a word embedding encoding of the input sequence $\mathrm{S}=\left\{\mathrm{S}_{0}, \mathrm{~S}_{1}, \mathrm{~S}_{2}, \ldots \mathrm{S}_{\mathrm{d}}\right\}$.

\section{(b) Embedding encoding}

Embedding is used to represent discrete variables as continuous vectors. It produces a dense vector with a fixed, arbitrary number of dimensions. Word embedding is one of the most popular representation of document vocabulary. The word embedding [37] representation can reveal many hidden relationships between phrases.

When using word embedding, the input sequence is converted into a numerical code based on Table 1, and the digitally encoded protein sequence is converted into dense vectors.

During this period, the sequence encoding layer generates a fixed-length feature represented by Encode $_{1}$ from the DNA binding protein sequence, using One-hot encoding or Word embedding encoding.

$$
\text { Encode }_{1}=\operatorname{Encode}\left(\mathrm{S}_{0}, \mathrm{~S}_{1}, \mathrm{~S}_{2}, \ldots \mathrm{S}_{\mathrm{n}}\right) \text {. }
$$

\subsubsection{Local feature learning}

A convolutional neural network was utilized to detect the functional domains of protein sequences. The local feature-leaning layer consisted of several blocks that performed convolution, batch-normalization, ReLU, and max-polling operation. Figure 4 presents the concrete structure.

This layer can use a One-hot encoding or word embedding encoding approach before the CNN structure. The specific network structure and parameter are discussed in Section 2.6. Experimental results using different encoding methods are presented in Section 3.1, 3.2 and 3.3. 
158

159

160

161

162

163

164

165

166

167

168

169

170

171

172

173

174

175

176

177

178

179

180

181

182

183

184

185

186

187

188

189

190

The local feature learning layer generates a representation of fixed length features which can be designated as Local $_{2}$.

$$
\text { Local }_{2}=\operatorname{Local}\left(\text { encode }_{0}, \text { encode }_{1}, \text { encode }_{2}, \ldots \text { encode }_{n}\right) .
$$

\subsubsection{Long-term context learning}

CNN-based prediction methods can get only the local characteristics of gene sequences. Since the gene sequence is long enough, it is desirable to use BI-LSTM to identify long-term dependencies. In our proposed model, the long-term context learning layer results in a characteristic representation of a fixed length, designated by Long_term 3 .

$$
\text { Long_term }_{3}=\text { Long_term }\left(\operatorname{local}_{0}, \operatorname{local}_{1}, \text { local }_{2}, \ldots \text { local }_{n}\right) \text {. }
$$

\subsubsection{Synthetic prediction}

The entry of the previous layer was concatenated into a vector and then went through a fully connected layer. Next, the hidden neurons "vote" on each of the labels, and the winner of that vote is the classification decision. The sigmoid function was used as the network activation function and cross-entropy function as the loss function. The fully connected layer generates output represented by $S_{\text {out }}$.

$$
\mathrm{S}_{\text {out }}=\text { Synthetic }\left(\text { long_term }_{0}, \text { long_term }_{1}, \text { long_term }_{2}, \ldots \text { long_term }_{n}\right) \text {. }
$$

\subsection{Model construction and evaluation}

Several validation methods were used to evaluate the performance of the proposed models. In a series of publications [1-6] in the field of Bioinformatics, k-fold cross-validation was widely used. In this paper, all experiments used 5-fold cross-validation to assess model performance on the PDB14189 dataset. Due to the relatively broad fluctuation range of the prediction results based on deep learning, the $\mathrm{k}$-fold $(\mathrm{k}=5)$ cross-validation was repeated five times. The average value was used in assessing the performance of the model. When evaluating model performance on the PDB14189 dataset, we follow the steps which illustrated in Figure 5:

Step 1. Take 11351 samples as the training set and take 2838 as the test set.

Step 2. Divide the 11,351 samples into two sections: (1) 10,215 samples were used for training, and (2) the remaining 1,136 samples were used for verification.

Step 3. Repeat $\mathrm{k}$-fold $(\mathrm{k}=5)$ cross-validation five times. The mean value was used to measure the performance of the model.

We conduct the independent test on the PDB2272 dataset as follows:

Step 1. Train the PDBP-Fusion model, which take $80 \%$ of the samples in PDB14189 as a training set and use the rest $(20 \%)$ as a validation set.

Step 2. Save the well-trained PDBP-Fusion model with the optimal parameter configuration, and then predict the PDB2272 independent dataset. 
191

192

193

194

195

196

197

198

199

200

201

202

203

204

205

206

207

208

209

210

211

212

213

214

215

216

217

218

219

220

221

222

223

224

Step 3. Compare the prediction results with other methods to evaluate model generalization.

Five evaluation indicators, including accuracy (ACC), precision (PRE), sensitivity (SE), specificity (SP), Matthew's Correlation Coefficient (MCC), were used as the performance measure. The various performance measures were defined as follows:

$$
\begin{aligned}
\mathrm{ACC}=\frac{(\mathrm{TP}+\mathrm{TN})}{(\mathrm{TP}+\mathrm{NP}+\mathrm{FP}+\mathrm{FN})} \\
\mathrm{PRE}=\frac{\mathrm{TP}}{(\mathrm{TP}+\mathrm{FP})} \\
\mathrm{SE}==\frac{\mathrm{TP}}{(\mathrm{TP}+\mathrm{FN})} \\
\mathrm{SP}=\frac{\mathrm{TN}}{(\mathrm{TN}+\mathrm{FP})} \\
\mathrm{MCC}=\frac{(\mathrm{TP} * \mathrm{TN}-\mathrm{FP} * \mathrm{FN})}{\sqrt{((\mathrm{TP}+\mathrm{FP}) *(\mathrm{TP}+\mathrm{FN}) *(\mathrm{TN}+\mathrm{FP}) *(\mathrm{TN}+\mathrm{FN}))}}
\end{aligned}
$$

TN, FN, TP, and FP represent the number of true negative, false negative, true positive, and false positive samples predicted. The area under the ROC (AUC) [38] is also used to evaluate prediction performance.

\subsection{Experimental parameter configuration}

The entire procedure was implemented based on the Keras framework. Complete codes, including the One-hot code, word-embedding code, CNN, Bi-LSTM, PDBP-CNN, and PDBP-Fusion code, are provided via http://119.45.144.26:8080/PDBP-Fusion/. Table 2. gives the detailed parameters of the proposed models.

\section{Results}

In this section, we first elaborate two series of comparative experiments based on different lengths of the sequence dataset. Next, we select other model parameters such as dropout ratio and convolution kernel size to obtain the optimal parameter configuration of the PDBP-Fusion model. Finally, we present the performance of PDBP-Fusion with other published studies on the same benchmark dataset PDB14189 and the independent dataset PDB2272.

\subsection{Performance comparison of PDBP-CNN models using One-hot encoding}

During the data processing phase, we selected different max length from 500 to 1000 to encode the DNA sequence. Then we evaluate the overall performance of the PDBP-CNN model based on One-hot encoding. When the maximum sequence length exceeded 1000, it became impossible to finish the 5-fold cross-validation experiment five times due to excessive memory consumption. Table 3 shows the experimental results.

Table 3 shows that convolutional neural networks can learn sophisticated features. The best performance ( $\mathrm{MCC}=64.69 \%$ and $\mathrm{ROC}-\mathrm{AUC}=90.29 \%$ ) was achieved with a sequence length equals 850 . However, model performance did not increase monotonically as maximum sequence lengths increased. The experimental results showed little difference when the maximum sequence length exceeds 700 under the same structural network model. 
225

226

227

228

229

230

231

232

233

234

235

236

237

238

239

240

241

242

243

244

245

246

247

248

249

250

251

252

253

254

255

256

257

258

259

260

\subsection{Performance comparison of PDBP-Fusion models using One-hot encoding}

Previously, the classic CNN network model was used for prediction, which does not obtain the longterm context dependencies in sequences. This subsection describes the use of PDBP-Fusion to evaluate performance. Table 4 presents the comparative experimental results.

In this series of experiments, the best performances (MCC of $66.1 \%$ and ROC-AUC of $90.83 \%$ ) were achieved. Experience has shown that the performance of the PDBP-Fusion model does not improve with increasing sequence length. When the sequence length is 700 , the optimum performance was achieved, after which it gradually decreased. Experiments show that the Bi-LSTM network can capture long-term dependencies even with a sequence length of less than 700 .

\subsection{Performance comparison of PDBP-Fusion models using word embedding}

In this section, we reported the model performances of PDBP-CNN and PDBP-Fusion based on word embedding encoding. We conducted two identical experiments with different sequence lengths that vary from 100 to 1000. After the sequence length exceeded 1000, it became impossible, as before, to complete the 5-fold cross-validation five times due to excessive GPU memory consumption. The best performances listed in Table 5 are each presented with their two best-archived results for all sequence lengths. Experiments have shown that the PDBP-Fusion approach can obtain all-round performance advantages superior to the PDBP-CNN method based on word embedding encoding.

\subsection{Model parameter selection and optimization}

Based on the comparative experiments in Section 3.1, 3.2 and 3.3, it is apparent that PDBP-CNN and PDBP-Fusion with the One-hot encoding approach showed better performance than the word embedding practice. Figure 6 shows that the PDBP-Fusion model with One-hot encoding obtained the best results $(\mathrm{MCC}=66.10 \%, \mathrm{AUC}=90.83 \%)$ and that the PDBP-CNN model with one-hot encoding obtained the second-best performance result $(\mathrm{MCC}=64.69 \%, \mathrm{AUC}=90.29 \%)$.

The best-performing architectures were then identified by varying the CNN convolution kernel width and the dropout ratio, as described in the following sections. The previous two best model performances were improved by tuning the network parameters.

\subsubsection{Selecting different dropout ratio $s$ in CNN}

As shown in Figure 7, the variation range of dropout parameters of the PDBP-Fusion model was $[0.1,0.2,0.3,0.4]$, whereas the other parameters remained unchanged. MCC and AUC both reached their optimal values when dropout ratio=0.3. In the same case, the optimal performance of the PDBP-CNN model was achieved when dropout ratio $=0.2$.

\subsubsection{Selecting different convolution kernels in CNN}

As shown in Figure 8, the convolution kernel size parameter of the PDBP-Fusion model varies in the range of $[5,7,9]$, whereas the other parameters remained unchanged. The model achieved optimal performance $(\mathrm{MCC}=66.10 \%, \mathrm{AUC}=90.83 \%)$ when the convolution kernel size $=9$. In the same case, the optimal performance of the PDBP-CNN model was reached when the convolution kernel size $=7$. 
270

271

272

273

274

275

276

277

278

279

280

281

282

283

284

285

286

287

288

289

290

291

292

293

294

295

296

The optimal models for PDBP-CNN and PDBP-Fusion were identified based on a series of experiments. Table 6 gives details of these performance results. The encoding approach and the network design parameters are listed for each.

\subsection{Performance comparison on the benchmark dataset}

In this section, we compare the performance of PDBP-Fusion with previously published methods such as DNABP [18], MsDBP [34] and StackDPPred [20] approach on the same benchmark dataset. The DNABP approach [18] combines various carefully selected manual features beyond the scope of this work. They rely on biological databases and require biological expertise. The PDBP-CNN and PDBP-Fusion methods are based only on the primary sequence and do not require manual feature extraction.

A comparison experiment based on StackDPPred [20] and One-hot encoding were carried out on the PDB14189 dataset. We first use One-hot for encoding an input sequence and then flatten the input vector. We entered these features into StackDPPred method and explored Base and Meta Classifiers. In order to find the base-classifiers to use in the first stage and the meta-classifier to use in the second stage of stacking framework, four different machine learning algorithms such as SVM, KNN, LogReg and RDF were explored. Figure 9 shows the StackDPPred prediction framework using One-hot encoding.

We optimize each classifier by cross-validation based on 50\% of the PDB14189 dataset. And we evaluate the trained model's performance on the remaining 50\% of the PDB14189.

(i) SVM: The best values of the base-classifier SVM parameters are $\mathrm{C}=1$ and $\gamma=0.0001$. Likewise, the best values of the parameters of the SVM, used as meta-classifier, are $\mathrm{C}=1$ and $\gamma=0.0001$.

(ii ) Logreg: In our implementation, we find $\mathrm{C}=0.0400$ results in the best accuracy.

(iii) RDF: In our implementation of the RDF ensemble learner, we have used bootstrap samples to construct 2000 trees in the forest.

(iv) $\mathrm{KNN}$ : In this work, the value of $\mathrm{k}$ is set to 6 , and all neighbours are weighted uniformly.

Table 7 indicates that the PDBP-Fusion methods achieved better performance than some random forest classifier models [18] with manually extracted features such as PSSM, PSSM-PP, PHY, etc. Our approach used the characteristics of deep learning and self-learning ability to identify DBPs based only on the sequences. Experimental results show that the performance of the PDBP-Fusion method was significantly improved compared with that of MsDBP. The MCC values for PDB-CNN and PDB-Fusion increased by at least $9.1 \%$ and $6.7 \%$ respectively. The AUC values increased by $2.8 \%$ and $2.2 \%$, respectively.

\subsection{Performance comparison on the independent test datasets}

The PDBP-Fusion model was then evaluated on the independent dataset (PDB2272) to verify its robustness. The comparison covered the proposed method with other advanced methods. Table 8 shows the experimental results.

In Table 8, the ACC value of PDBP-Fusion on PDB2272 exceeds other prediction methods. The ACC of PDBP-Fusion is $77.77 \%$, which is $16.7 \%$ higher than the ACC of MsDBP (77.77\% vs $66.99 \%$ ). From the perspective of model stability, the MCC of PDBP-Fusion is 0.5665 , which is $66.8 \%$ higher than the

PeerJ reviewing PDF | (2020:08:52191:1:1:NEW 3 Mar 2021) 
297

298

299

300

301

302

303

304

305

306

307

308

309

310

311

312

313

314

315

316

317

318

319

320

321

322

323

324

325

326

327

328

329

330

331

332

MCC of MsDBP. It demonstrates that the PDBP-Fusion model has got superior performance and model robustness on the PDB2272 independent dataset.

\subsection{Web server for PDBP-Fusion}

Many advanced methods [39]-[42] provide an available Web server and prediction tool for users to predict DBPs online. We also offer a Web server at http://119.45.144.26:8080/PDBP-Fusion/. Additionally, we provide all the steps to get the predicted results for convenience.

Step 1. Click the link, and you will see the index page is shown in Fig. 10.

Step 2. Click the "Download" link, and you can download the benchmark dataset, independent dataset, and the codes.

Step 3. Either type or copy and paste the protein sequence into the input box in Fig. 10, Click the "Predict" button to see the predicted results.

\section{Conclusions}

The CNN based method alone is not accurate enough in predicting DBPs from DNA sequences. In this his study, the CNN network was used to find suitable local features, and Bi-LSTM was used to capture long-term dependencies among DNA sequences. It is preferable to predict DBPs by merging the local features obtained by CNN with the long-term context-dependent features captured by Bi-LSTM. Some existing methods have also combined CNN and LSTM to predict DBPs, but they completed the preprocessing of gene sequences based on word embedding. The One-hot encoding method used in this study is straightforward and more efficient than word embedding. It is also necessary to consider designing a convolutional neural network reasonably to match the DNA sequence data's characteristics and distribution.

The PDBP-Fusion method proposed in this paper has demonstrated its significance on the PDB14189 benchmark dataset and its performance relative to the outcomes of existing methods on the PDB2272 independent dataset. The proposed method showed remarkably higher generalization ability compared with existing methods. Furthermore, this study suggests that the fusion approach, combining local features and long-term dependencies, will be necessary for sequence-based tasks in genomics. It also provides a solution to other sequential prediction problems.

\section{Data Availability}

The datasets and source codes for this study are freely available to the academic community at https://github.com/hfuulgb/PDBP-Fusion.

\section{Competing Interests}

The authors declare there are no competing interests.

\section{References}

[1] W. M. Krajewska, "Regulation of transcription in eukaryotes by DNA-binding proteins," Int. J. Biochem., vol. 24, no. 12, pp. 1885-1898, 1992.

[2] N. M. Luscombe, S. E. Austin, H. M. Berman, and J. M. Thornton, "An overview of the structures of protein-DNA complexes,” Genome Biol., vol. 1, no. 1, pp. 1-37, 2000. 
333

334

335

336

337

338

339

340

341

342

343

344

345

346

347

348

349

350

351

352

353

354

355

356

357

358

359

360

361

362

363

364

365

366

367

368

369

370

371

372

373

374

375

376

377

378

379

380

381

382

383

[3] W. Lou, X. Wang, F. Chen, Y. Chen, B. Jiang, and H. Zhang, "Sequence based prediction of DNA-binding proteins based on hybrid feature selection using random forest and Gaussian naïve Bayes," PLoS One, vol. 9, no. 1, pp. 1-10, 2014.

[4] Y. D. Cai and S. L. Lin, "Support vector machines for predicting rRNA-, RNA-, and DNAbinding proteins from amino acid sequence," Biochim. Biophys. Acta - Proteins Proteomics, vol. 1648, no. 1-2, pp. 127-133, 2003.

[5] N. Bhardwaj, R. E. Langlois, G. Zhao, and H. Lu, "Kernel-based machine learning protocol for predicting DNA-binding proteins," Nucleic Acids Res., vol. 33, no. 20, pp. 6486-6493, 2005.

[6] X. Yu, J. Cao, Y. Cai, T. Shi, and Y. Li, "Predicting rRNA-, RNA-, and DNA-binding proteins from primary structure with support vector machines," J. Theor. Biol., vol. 240, no. 2, pp. 175184, 2006.

[7] M. Kumar, M. M. Gromiha, and G. P. S. Raghava, "Identification of DNA-binding proteins using support vector machines and evolutionary profiles," BMC Bioinformatics, vol. 8, pp. 1-10, 2007.

[8] C. Zou, J. Gong, and H. Li, "An improved sequence based prediction protocol for DNA-binding proteins using SVM and comprehensive feature analysis," BMC Bioinformatics, vol. 14, 2013.

[9] Y. Zhang et al., "NewDNA-Prot: Prediction of DNA-binding proteins by employing support vector machine and a comprehensive sequence representation," Comput. Biol. Chem., vol. 52, pp. 51-59, 2014.

[10] B. Liu et al., "IDNA-Prot|dis: Identifying DNA-binding proteins by incorporating amino acid distance-pairs and reduced alphabet profile into the general pseudo amino acid composition," PLoS One, vol. 9, no. 9, 2014.

[11] Y. Fang, Y. Guo, Y. Feng, and M. Li, "Predicting DNA-binding proteins: Approached from Chou's pseudo amino acid composition and other specific sequence features," Amino Acids, vol. 34, no. 1, pp. 103-109, 2008.

[12] E. W. Stawiski, L. M. Gregoret, and Y. Mandel-Gutfreund, "Annotating nucleic acid-binding function based on protein structure," J. Mol. Biol., vol. 326, no. 4, pp. 1065-1079, 2003.

[13] Y. Ofran, V. Mysore, and B. Rost, "Prediction of DNA-binding residues from sequence," Bioinformatics, vol. 23, no. 13, pp. 347-353, 2007.

[14] H. Tjong and H. X. Zhou, "DISPLAR: An accurate method for predicting DNA-binding sites on protein surfaces," Nucleic Acids Res., vol. 35, no. 5, pp. 1465-1477, 2007.

[15] M. B. Carson, R. Langlois, and H. Lu, "NAPS: A residue-level nucleic acid-binding prediction server," Nucleic Acids Res., vol. 38, no. SUPPL. 2, pp. 431-435, 2010.

[16] J. Wu et al., "Prediction of DNA-binding residues in proteins from amino acid sequences using a random forest model with a hybrid feature," Bioinformatics, vol. 25, no. 1, pp. 30-35, 2009.

[17] K. K. Kumar, G. Pugalenthi, and P. N. Suganthan, "Dna-prot: Identification of dna binding proteins from protein sequence information using random forest," J. Biomol. Struct. Dyn., vol. 26, no. 6, pp. 679-686, 2009.

[18] X. Ma, J. Guo, and X. Sun, "DNABP: Identification of DNA-binding proteins based on feature selection using a random forest and predicting binding residues," PLoS One, vol. 11, no. 12, pp. 120, 2016.

[19] M. S. Rahman, S. Shatabda, S. Saha, M. Kaykobad, and M. S. Rahman, "DPP-PseAAC: A DNAbinding protein prediction model using Chou's general PseAAC," J. Theor. Biol, vol. 452, pp. 22-34, 2018.

[20] A. Mishra, P. Pokhrel, and M. T. Hoque, "StackDPPred: A stacking based prediction of DNAbinding protein from sequence," Bioinformatics, vol. 35, no. 3, pp. 433-441, 2019.

[21] J. Wang, H. Zheng, Y. Yang, W. Xiao, and T. Liu, "PredDBP-Stack: Prediction of DNA-Binding Proteins from HMM Profiles using a Stacked Ensemble Method," Biomed Res. Int., vol. 2020, 2020.

[22] Q. Zhang, P. Liu, Y. Han, Y. Zhang, X. Wang, and B. Yu, "StackPDB: predicting DNA-binding proteins based on XGB-RFE feature optimization and stacked ensemble classifier," bioRxiv, $\mathrm{p}$. 2020.08.24.264267, 2020 . 
384

385

386

387

388

389

390

391

392

393

394

395

396

397

398

399

400

401

402

403

404

405

406

407

408

409

410

411

412

413

414

415

416

417

418

419

420

421

422

423

424

425

426

427

428

429

430

431

432

433

434

[23] R. Collobert, J. Weston, L. Bottou, M. Karlen, K. Kavukcuoglu, and P. Kuksa, "Natural language processing (almost) from scratch," J. Mach. Learn. Res., vol. 12, no. March 2011, pp. 2493-2537, 2011.

[24] A. Krizhevsky, I. Sutskever, and G. E. Hinton, "ImageNet classification with deep convolutional neural networks," Commun. ACM, vol. 60, no. 6, pp. 84-90, 2017.

[25] H. Tayara, K. G. Soo, and K. T. Chong, "Vehicle Detection and Counting in High-Resolution Aerial Images Using Convolutional Regression Neural Network," IEEE Access, vol. 6, pp. 22202230, 2017.

[26] H. Tayara and K. T. Chong, "Object detection in very high-resolution aerial images using onestage densely connected feature pyramid network," Sensors (Switzerland), vol. 18, no. 10, 2018.

[27] B. Alipanahi, A. Delong, M. T. Weirauch, and B. J. Frey, "Predicting the sequence specificities of DNA- and RNA-binding proteins by deep learning," Nat. Biotechnol., vol. 33, no. 8, pp. 831-838, 2015.

[28] H. Zeng, M. D. Edwards, G. Liu, and D. K. Gifford, "Convolutional neural network architectures for predicting DNA-protein binding," Bioinformatics, vol. 32, no. 12, pp. i121-i127, Jun. 2016.

[29] J. Zhou, Q. Lu, R. Xu, L. Gui, and H. Wang, "CNNsite: Prediction of DNA-binding residues in proteins using Convolutional Neural Network with sequence features," Proc. - 2016 IEEE Int. Conf. Bioinforma. Biomed. BIBM 2016, no. December, pp. 78-85, 2017.

[30] S. Shadab, M. T. A. Khan, N. A. Neezi, S. Adilina, and S. Shatabda, "DeepDBP: Deep Neural Networks for Identification of DNA-binding Proteins," bioRxiv, p. 829432, 2019.

[31] Y. Zhang, S. Qiao, S. Ji, and Y. Li, "DeepSite: bidirectional LSTM and CNN models for predicting DNA-protein binding," Int. J. Mach. Learn. Cybern., no. July, 2019.

[32] Y. H. Qu, H. Yu, X. J. Gong, J. H. Xu, and H. S. Lee, "On the prediction of DNA-binding proteins only from primary sequences: A deep learning approach," PLoS One, vol. 12, no. 12, pp. 1-18, 2017.

[33] S. Hu, R. Ma, and H. Wang, "An improved deep learning method for predicting DNA-binding proteins based on contextual features in amino acid sequences," PLoS One, vol. 14, no. 11, pp. 1$21,2019$.

[34] X. Du, Y. Diao, H. Liu, and S. Li, "MsDBP: Exploring DNA-Binding Proteins by Integrating Multiscale Sequence Information via Chou's Five-Step Rule," J. Proteome Res., vol. 18, no. 8, pp. 3119-3132, 2019.

[35] B. Liu, J. Xu, S. Fan, R. Xu, J. Zhou, and X. Wang, "PseDNA-Pro: DNA-binding protein identification by combining chou's PseAAC and Physicochemical distance transformation," Mol. Inform., vol. 34, no. 1, pp. 8-17, 2015.

[36] L. Wei, J. Tang, and Q. Zou, "Local-DPP: An improved DNA-binding protein prediction method by exploring local evolutionary information," Inf. Sci. (Ny)., vol. 384, pp. 135-144, 2017.

[37] R. Collobert, J. Weston, L. Bottou, M. Karlen, K. Kavukcuoglu, and P. Kuksa, "Natural language processing (almost) from scratch," J. Mach. Learn. Res., vol. 12, no. 20, pp. 2493-2537, May 2011.

[38] T. Fawcett, "ROC Graphs: Notes and Practical Considerations for Data Mining Researchers ROC Graphs : Notes and Practical Considerations for Data Mining Researchers," HP Inven., p. 27, 2003.

[39] W. Chen, P. Feng, H. Yang, H. Ding, H. Lin, and K. C. Chou, "IRNA-AI: Identifying the adenosine to inosine editing sites in RNA sequences," Oncotarget, vol. 8, no. 3, pp. 4208-4217, 2017.

[40] W. R. Qiu, B. Q. Sun, X. Xiao, Z. C. Xu, J. H. Jia, and K. C. Chou, "iKcr-PseEns: Identify lysine crotonylation sites in histone proteins with pseudo components and ensemble classifier," Genomics, vol. 110, no. 5, pp. 239-246, 2018.

[41] X. Cheng, W. Z. Lin, X. Xiao, and K. C. Chou, "PLoc-bal-mAnimal: Predict subcellular localization of animal proteins by balancing training dataset and PseAAC," Bioinformatics, vol. 35, no. 3, pp. 398-406, 2019.

Peer) reviewing PDF | (2020:08:52191:1:1:NEW 3 Mar 2021) 
435 [42] K. C. Chou, X. Cheng, and X. Xiao, "pLoc_bal-mHum: Predict subcellular localization of human

436

437 proteins by PseAAC and quasi-balancing training dataset," Genomics, vol. 111, no. 6, pp. 1274$1282,2019$.

438 
Table $\mathbf{1}$ (on next page)

Amino acid encoder. 
Table 1. Amino acid encoder.

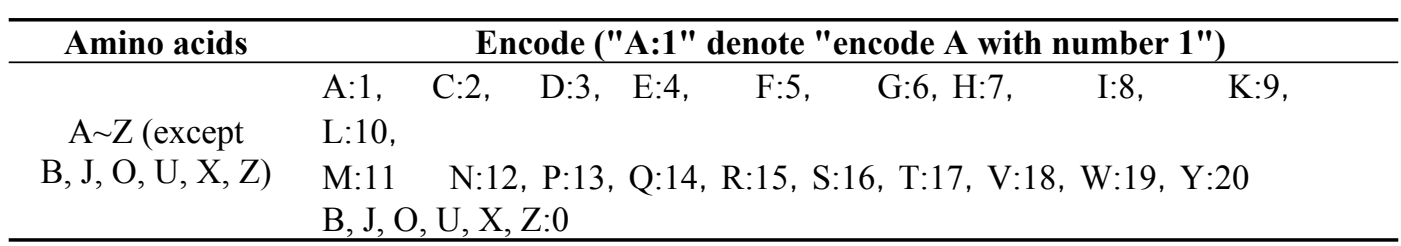

2 
Table 2 (on next page)

Parameters details of the proposed models.

"Len" denotes the input sequence max length. 
Table 2. Parameters details of the proposed models.

\begin{tabular}{|c|c|c|c|c|}
\hline Layers & PDBP-CNN & Output shape & PDBP-Fusion & Output shape \\
\hline 1 & One-hot encoding & Len*20 & One-hot encoding & Len $* 20$ \\
\hline 2 & $\begin{array}{c}\text { Convolution } 1 \\
(\text { kernel }=7, \text { stride }=1)\end{array}$ & Len *64 & $\begin{array}{c}\text { Convolution } 1 \\
(\text { kernel }=9, \text { stride }=1)\end{array}$ & Len $* 64$ \\
\hline 3 & $\begin{array}{c}\text { Max-polling1 } \\
(\text { kernel }=2)\end{array}$ & $(\operatorname{Len} / 2) * 64$ & $\begin{array}{c}\text { Max-polling1 } \\
(\text { kernel=2) }\end{array}$ & $(\operatorname{Len} / 2) * 64$ \\
\hline 4 & $\begin{array}{c}\text { Convolution } 2 \\
(\text { kernel }=7, \text { stride }=1)\end{array}$ & $(\operatorname{Len} / 2) * 64$ & $\begin{array}{c}\text { Convolution } 2 \\
(\text { kernel }=9, \text { stride }=1)\end{array}$ & $(\operatorname{Len} / 2) * 64$ \\
\hline 5 & $\begin{array}{c}\text { Max-polling2 } \\
(\text { kernel }=2)\end{array}$ & $(\operatorname{Len} / 4) * 64$ & $\begin{array}{c}\text { Max-polling2 } \\
(\text { kernel }=2)\end{array}$ & $(\operatorname{Len} / 4) * 64$ \\
\hline 6 & $\begin{array}{c}\text { Convolution3 } \\
(\text { kernel }=7, \text { stride }=1)\end{array}$ & $(\operatorname{Len} / 2) * 64$ & Bi- $\operatorname{LSTM}(32)$ & $150 * 64$ \\
\hline 7 & $\begin{array}{c}\text { Max-polling3 } \\
(\text { kernel }=2)\end{array}$ & $(\operatorname{Len} / 8) * 64$ & Dense(128) & 128 \\
\hline 8 & Dense (128) & 128 & Dense(2) & 2 \\
\hline 9 & Dense (2) & 2 & & \\
\hline
\end{tabular}


Table 3(on next page)

Quantitative results of the PDB-CNN method with different maximum sequence lengths. 
1 Table 3. Quantitative results of the PDB-CNN method with different maximum sequence lengths.

\begin{tabular}{ccccccc}
\hline $\boldsymbol{L}_{\boldsymbol{m a x}}$ & ACC (\%) & SE (\%) & PRE (\%) & SP (\%) & MCC (\%) & AUC (\%) \\
\hline 100 & 76.51 & 73.83 & 83.53 & 69.43 & 54.05 & 85.26 \\
150 & 78.85 & 76.92 & 83.29 & 74.37 & 58.27 & 87.21 \\
200 & 79.48 & 77.9 & 83.28 & 75.64 & 59.55 & 88.02 \\
250 & 80.38 & 78.61 & 84.17 & 76.56 & 61.21 & 88.71 \\
300 & 80.72 & 79.42 & 83.70 & 77.71 & 61.90 & 89.06 \\
350 & 81.04 & 77.95 & 87.32 & 74.71 & 62.86 & 89.42 \\
400 & 81.28 & 78.34 & 87.39 & 75.11 & 63.40 & 89.7 \\
450 & 81.52 & 79.21 & 86.20 & 76.80 & 63.59 & 89.94 \\
500 & 81.55 & 78.72 & 87.36 & 75.68 & 63.89 & 90.00 \\
550 & 81.32 & 78.52 & 87.21 & 75.38 & 63.46 & 89.86 \\
600 & 81.88 & 80.64 & 84.67 & 79.05 & 64.21 & 90.18 \\
650 & 81.74 & 80.58 & 84.49 & 78.97 & 63.97 & 90.12 \\
700 & 81.79 & 78.22 & 88.83 & 74.68 & 64.42 & 90.13 \\
750 & 81.68 & 79.04 & 87.07 & 76.24 & 64.08 & 89.92 \\
800 & 81.71 & 78.33 & $\mathbf{8 8 . 4 4}$ & 74.92 & 64.28 & 90.11 \\
850 & 82.02 & 79.25 & 87.49 & 76.50 & $\mathbf{6 4 . 6 9}$ & $\mathbf{9 0 . 2 9}$ \\
900 & 81.94 & 79.09 & 87.51 & 76.32 & 64.53 & 90.13 \\
950 & 81.91 & 79.50 & 86.74 & 77.03 & 64.44 & 90.24 \\
1000 & $\mathbf{8 2 . 0 4}$ & 80.33 & 85.40 & $\mathbf{7 8 . 6 5}$ & 64.43 & 90.05 \\
$>1000$ & -- & -- & -- & -- & -- & -- \\
\hline
\end{tabular}

2 
Table 4 (on next page)

Quantitative results of the PDBP-Fusion method with different maximum sequence lengths. 
Table 4. Quantitative results of the PDBP-Fusion method with different maximum sequence lengths.

\begin{tabular}{ccccccc}
\hline $\boldsymbol{L}_{\boldsymbol{m a x}}$ & ACC (\%) & SE (\%) & PRE (\%) & SP (\%) & MCC (\%) & AUC (\%) \\
\hline 100 & 77.28 & 74.53 & 83.99 & 70.50 & 55.42 & 85.79 \\
150 & 78.91 & 76.62 & 84.24 & 73.53 & 58.57 & 87.36 \\
200 & 80.08 & 77.88 & 84.77 & 75.35 & 60.71 & 88.33 \\
250 & 80.74 & 79.51 & 83.56 & 77.89 & 61.9 & 88.96 \\
300 & 81.44 & 79.57 & 85.25 & 77.60 & 63.33 & 89.50 \\
350 & 82.27 & 80.39 & 85.8 & 78.71 & 64.83 & 90.01 \\
400 & 81.70 & 79.48 & 86.38 & 76.98 & 64.12 & 90.11 \\
450 & 82.30 & 79.36 & $\mathbf{8 7 . 8 4}$ & 76.71 & 65.15 & 90.37 \\
500 & 82.50 & 79.58 & 87.95 & 77.01 & 65.54 & 90.43 \\
550 & 82.16 & 79.97 & 86.56 & 77.72 & 64.90 & 90.37 \\
600 & 82.56 & 80.87 & 85.90 & $\mathbf{7 9 . 1 8}$ & 65.50 & 90.61 \\
650 & 82.81 & 80.84 & 86.56 & 79.03 & 66.02 & 90.7 \\
700 & $\mathbf{8 2 . 8 1}$ & $\mathbf{8 1 . 0 2}$ & 86.45 & 79.13 & $\mathbf{6 6 . 1}$ & $\mathbf{9 0 . 8 3}$ \\
750 & 82.66 & 80.51 & 86.82 & 78.45 & 65.8 & 90.65 \\
800 & 82.7 & 79.99 & 87.8 & 77.56 & 65.95 & 90.74 \\
850 & 82.71 & 80.51 & 86.93 & 78.44 & 65.89 & 90.73 \\
900 & 82.63 & 80.06 & 87.61 & 77.61 & 65.85 & 90.69 \\
$>900$ & -- & -- & -- & -- & -- & - \\
\hline
\end{tabular}




\section{Table 5 (on next page)}

PDBP-Fusion model performance using a word embedding encoding on the PDB14189 dataset.

${ }^{1}$ PDBP-Fusion model: (length $=800$, word embedding encoding, 64 convolution kernels).

${ }^{2}$ PDBP-Fusion model: (length $=800$, word embedding encoding, 32 convolution kernels). 
1 Table 5. PDBP-Fusion model performance using a word embedding encoding on the PDB14189 dataset.

\begin{tabular}{cccccc}
\hline Methods & ACC (\%) & SE (\%) & SP (\%) & MCC (\%) & AUC (\%) \\
\hline PDBP-Fusion $^{\mathbf{1}}$ & 81.01 & 78.48 & 81.58 & 62.0 & 89.03 \\
PDBP-Fusion $^{2}$ & 79.40 & 83.60 & 75.15 & 59.1 & 87.81 \\
\hline
\end{tabular}

21 PDBP-Fusion model: (length $=800$, word embedding encoding, 64 convolution kernels).

32 PDBP-Fusion model: (length $=800$, word embedding encoding, 32 convolution kernels). 


\title{
Table 6 (on next page)
}

Peak performance of PDBP-CNN and PDBP-Fusion models on the PDB14189 dataset.

\begin{abstract}
${ }^{1}$ PDBP-CNN model: The maximum length is 850 . The convolution layer has three layers, the convolution kernel is $\left(7^{*} 1\right)$, the maximum pooling size is $(2,1)$, and the total connection layer has 128 nodes. The dropout rate is set to 0.2 .

${ }^{2}$ PDBP-Fusion model: The maximum length is 700 . The convolution layer has two layers, the convolution kernel is $(9 * 1)$, the maximum pooling size is $(2,1)$, the number of cells in Bi-LSTM is set to $16 * 2$, and the total connection layer has 128 nodes. The dropout rate is set to 0.3 .
\end{abstract}


Table 6. Peak performance of PDBP-CNN and PDBP-Fusion models on the PDB14189 dataset.

\begin{tabular}{cccccc}
\hline Methods & ACC (\%) & SE (\%) & SP (\%) & MCC (\%) & AUC (\%) \\
\hline PDBP-CNN $^{\mathbf{1}}$ & $82.02 \pm 1.22$ & $87.49 \pm 4.12$ & $76.50 \pm 5.66$ & $64.69 \pm 1.87$ & $90.29 \pm 0.51$ \\
PDBP-Fusion $^{2}$ & $82.81 \pm 1.30$ & $86.45 \pm 4.59$ & $79.13 \pm 5.81$ & $66.1 \pm 2.04$ & $90.83 \pm 0.57$ \\
\hline
\end{tabular}

$2{ }^{1}$ PDBP-CNN model: The maximum length is 850 . The convolution layer has three layers, the convolution kernel is $(7 * 1)$, the maximum pooling size is $3(2,1)$, and the total connection layer has 128 nodes. The dropout rate is set to 0.2 .

42 PDBP-Fusion model: The maximum length is 700 . The convolution layer has two layers, the convolution kernel is ( $9 * 1)$, the maximum pooling size is 5 (2,1), the number of cells in Bi-LSTM is set to $16^{*} 2$, and the total connection layer has 128 nodes. The dropout rate is set to 0.3 . 


\section{Table 7 (on next page)}

Comparison of the proposed model with other methods on the PDB14189 dataset.

${ }^{1}$ DNABP method which using RF classifier and various features[18].

${ }^{2}$ StackDPPred(One-hot) method using StackDPPred and One-hot encoding [31]. 
Table 7. Comparison of the proposed model with other methods on the PDB14189 dataset.

\begin{tabular}{|c|c|c|c|c|c|}
\hline Methods & $\operatorname{ACC}(\%)$ & SE $(\%)$ & SP (\%) & $\operatorname{MCC}(\%)$ & $\operatorname{AUC}(\%)$ \\
\hline MsDBP & 80.29 & 80.87 & 79.72 & 60.61 & 88.31 \\
\hline $\mathrm{PSSM}^{1}$ & 79.62 & 76.02 & 83.21 & 59.4 & -- \\
\hline PSSM-PP ${ }^{1}$ & 81.69 & 78.92 & 84.45 & 63.5 & -- \\
\hline $\mathrm{PHY}^{1}$ & 77.65 & 73.54 & 81.76 & 55.5 & -- \\
\hline PSSM-PP+BP_NBP1 & 83.68 & 81.01 & 86.34 & 67.4 & -- \\
\hline PSSM-PP+PHY ${ }^{1}$ & 82.67 & 79.95 & 85.39 & 65.4 & -- \\
\hline $\mathrm{BP} \pm \mathrm{NBP}+\mathrm{PHY}^{1}$ & 80.40 & 76.88 & 83.92 & 60.9 & -- \\
\hline ALL features $^{1}$ & 84.64 & 82.23 & 87.06 & 70.6 & -- \\
\hline 64 Optimal features ${ }^{1}$ & 86.90 & 83.76 & 90.03 & 72.7 & -- \\
\hline StackDPPred(One-hot) ${ }^{2}$ & 76.00 & 79.27 & 72.71 & 52.10 & 83.18 \\
\hline PDBP-CNN & 82.02 & 87.49 & 76.50 & 64.69 & 90.29 \\
\hline PDBP-Fusion & 82.81 & 86.45 & 79.13 & 66.1 & 90.83 \\
\hline
\end{tabular}

$2 \quad{ }^{1}$ DNABP method which using RF classifier and various features [18].

$3 \quad{ }^{2}$ StackDPPred(One-hot) method using StackDPPred and One-hot encoding [31].

4 
Table 8(on next page)

Comparison of various machine learning methods on the PDB2272 dataset. 
Table 8 Comparison of various machine learning methods on the PDB2272 dataset.

\begin{tabular}{cccccc}
\hline Methods & ACC (\%) & SE (\%) & SP (\%) & MCC (\%) & AUC (\%) \\
\hline Qu et al. [47] & 48.33 & 49.07 & 48.31 & -3.34 & 47.76 \\
Local-DPP [21] & 50.57 & 58.72 & 8.76 & 4.56 & -- \\
PseDNA-Pro [20] & 61.88 & 59.90 & 75.28 & 24.30 & - \\
DPP-PseAAC [19] & 58.10 & 59.10 & 56.63 & 16.25 & 61.00 \\
MsDBP [45] & 66.99 & 66.42 & 70.69 & 33.97 & 73.83 \\
PDBP-Fusion & $\mathbf{7 7 . 7 7}$ & $\mathbf{7 3 . 3 1}$ & 66.85 & $\mathbf{5 6 . 6 5}$ & $\mathbf{8 5 . 3 9}$ \\
\hline
\end{tabular}

2 
Figure 1

Architecture of the proposed PDBP-Fusion model.

\section{Synthetic prediction layer}

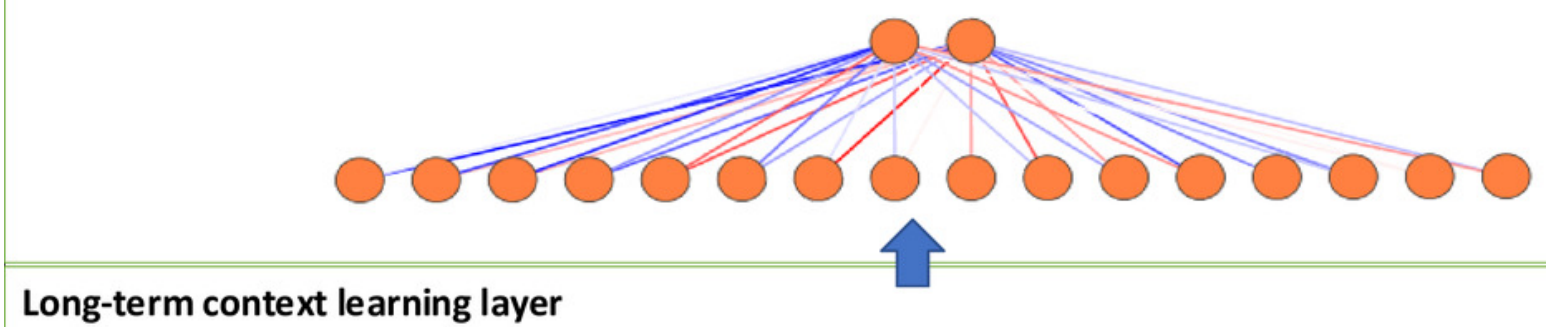

Local feature learning layer
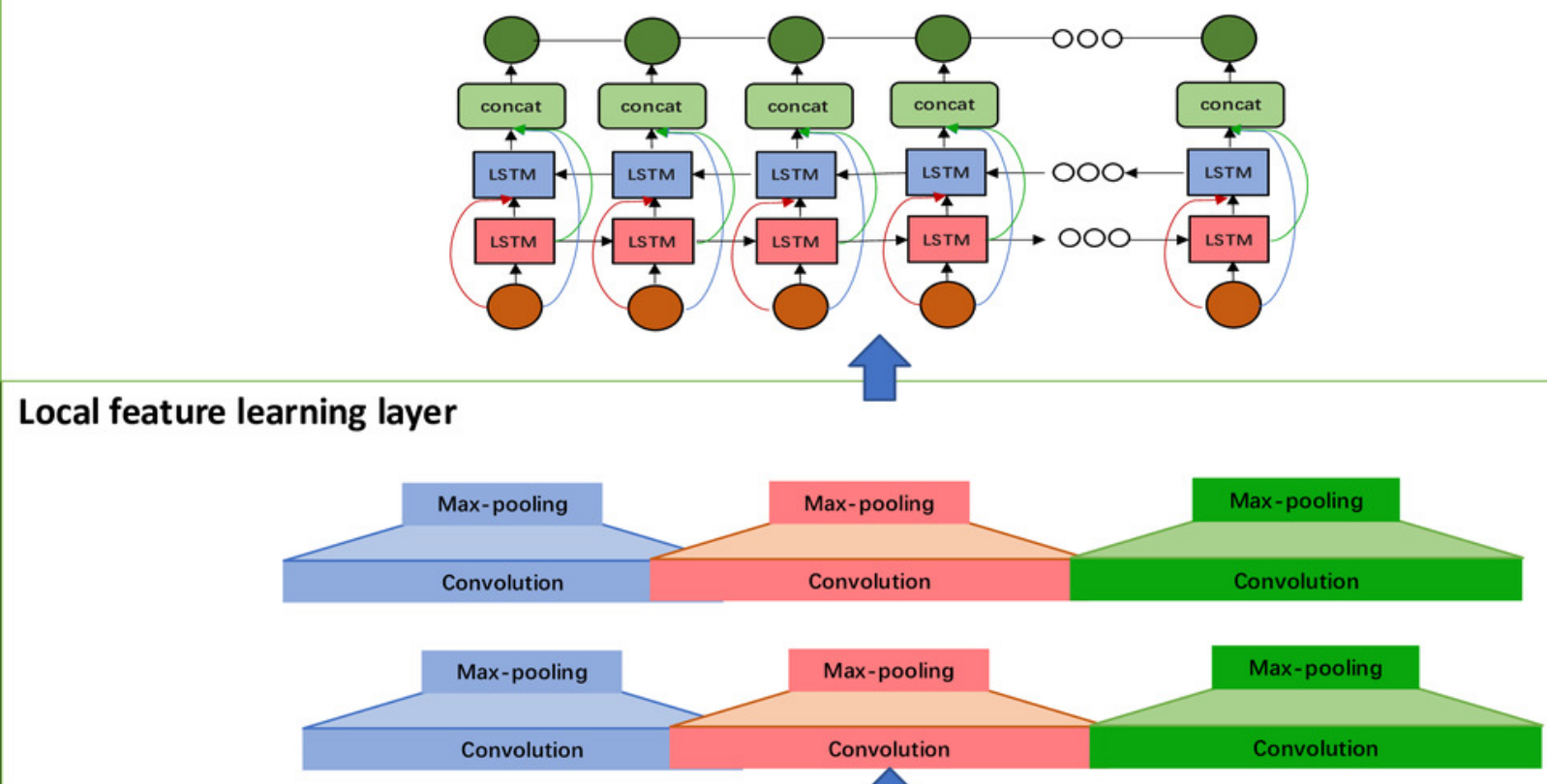

\section{Sequence encoding layer}

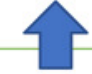

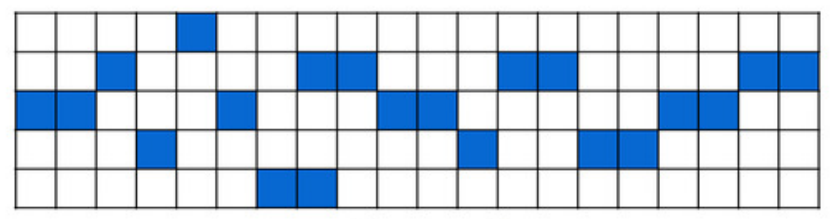

One-hot encoding

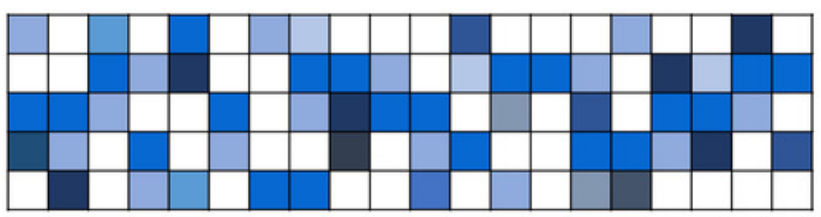

Embedding encoding

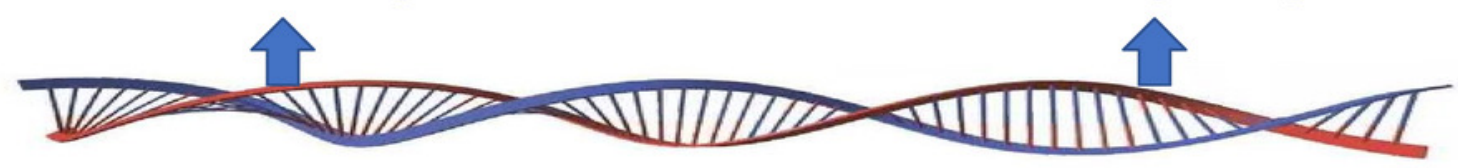


Figure 2

Statistical graph of DNA sequence length distribution in the PDB14189 dataset.

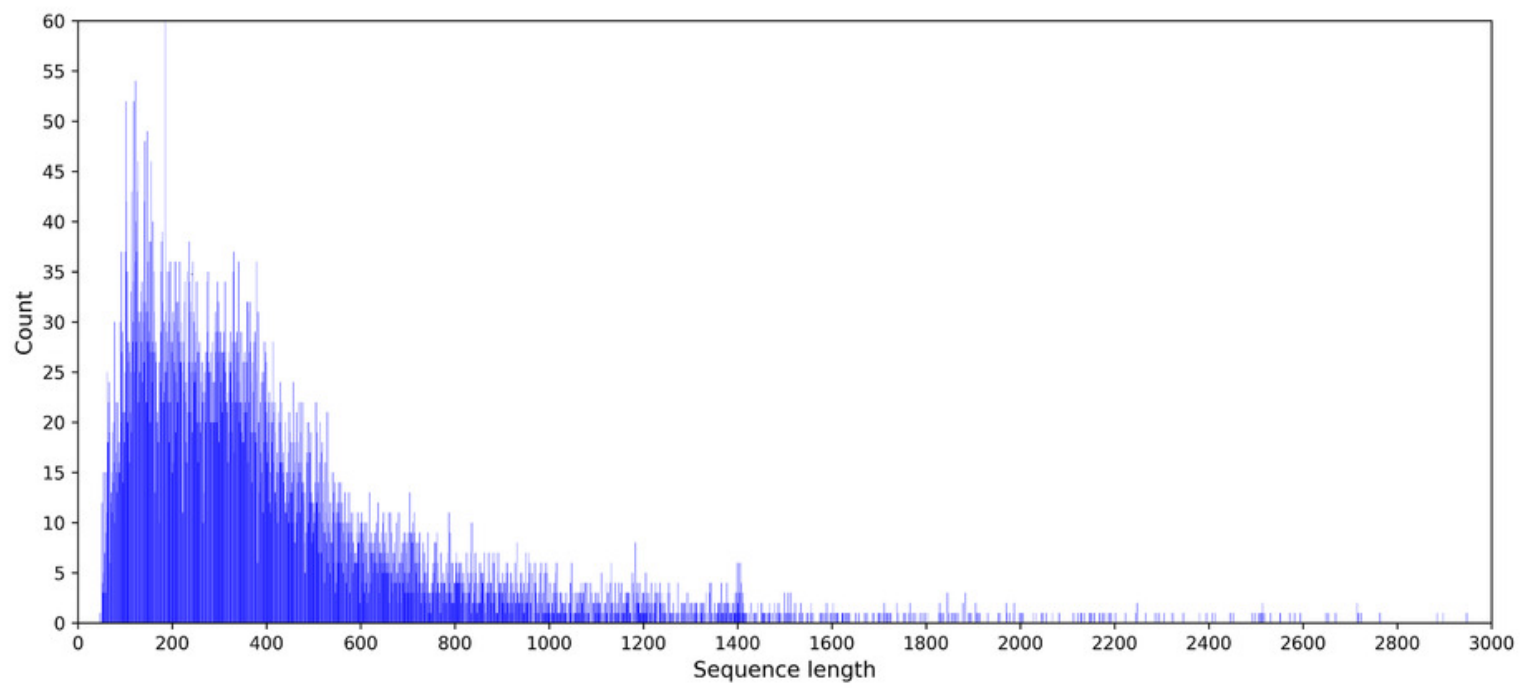


Figure 3

Coding diagram of (a) One-hot encoding and (b) word embedding encoding.

Sequence $\mathbf{S}=\left\{\mathbf{S}_{0}, \mathbf{S}_{1}, \mathbf{S}_{2}, \ldots \mathbf{S}_{n}\right\}$, length $=\mathbf{d}$.

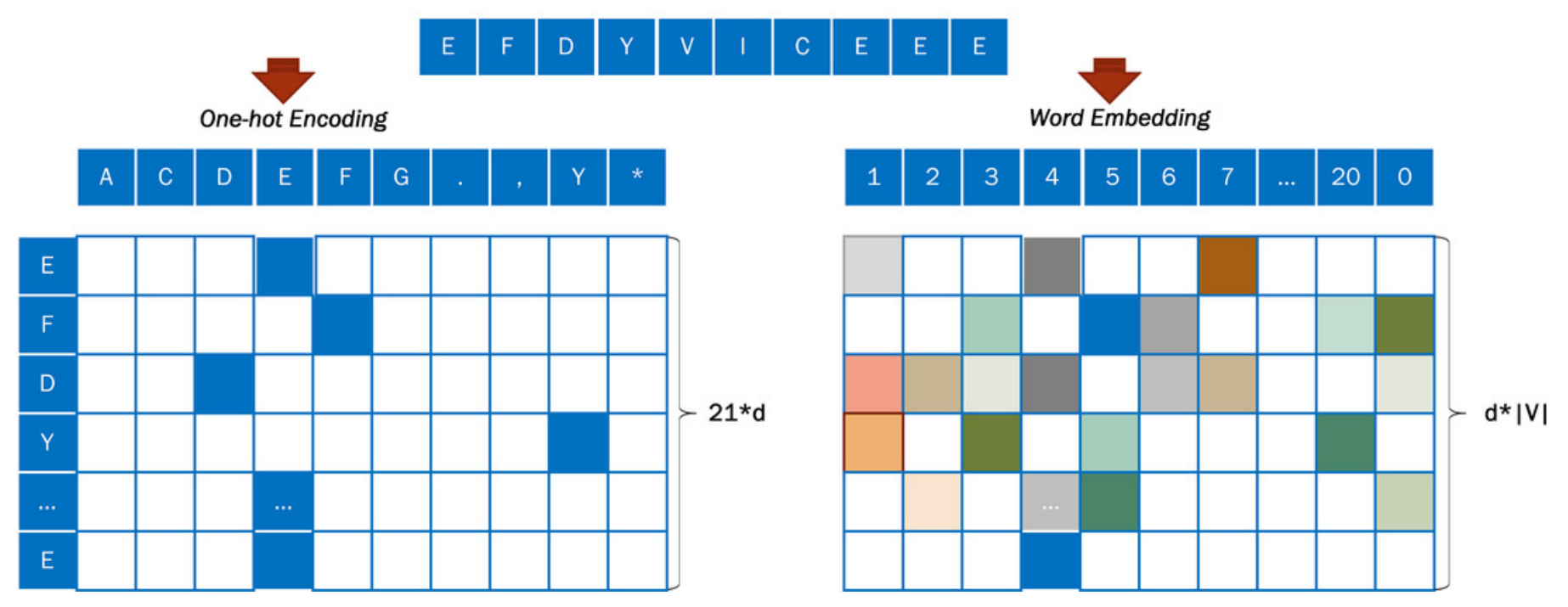


Figure 4

CNN network structure.

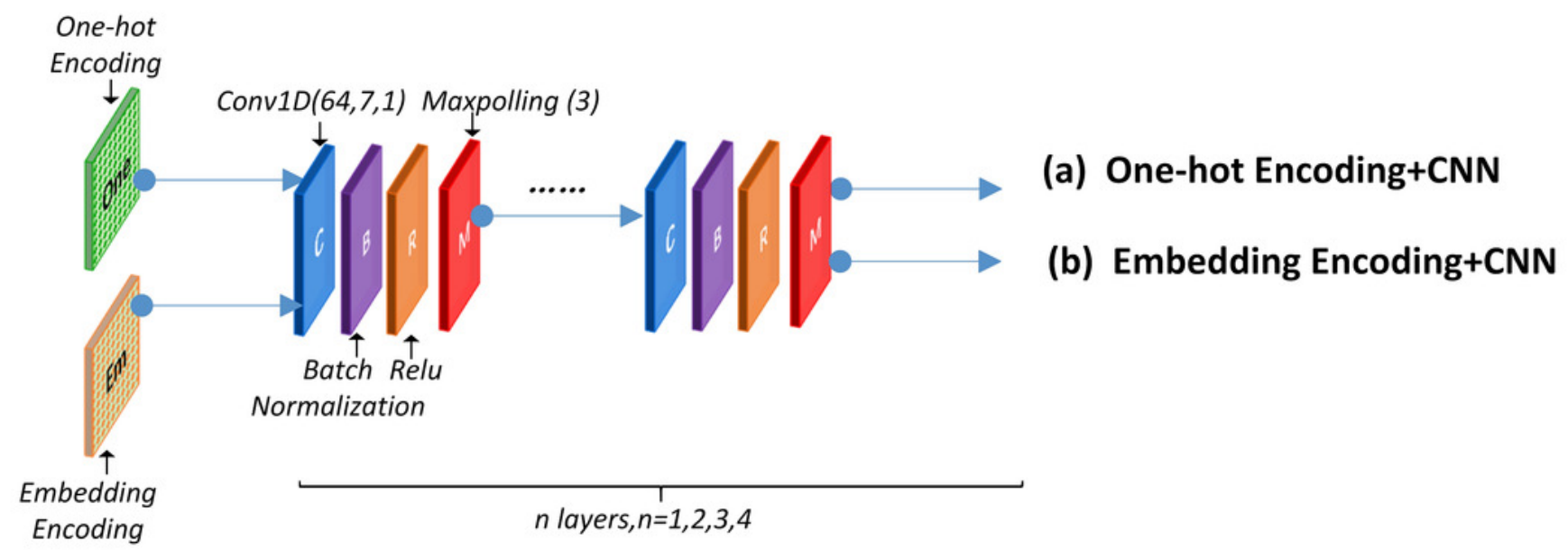


Figure 5

Model evaluation on benchmark datasets PDB14189.

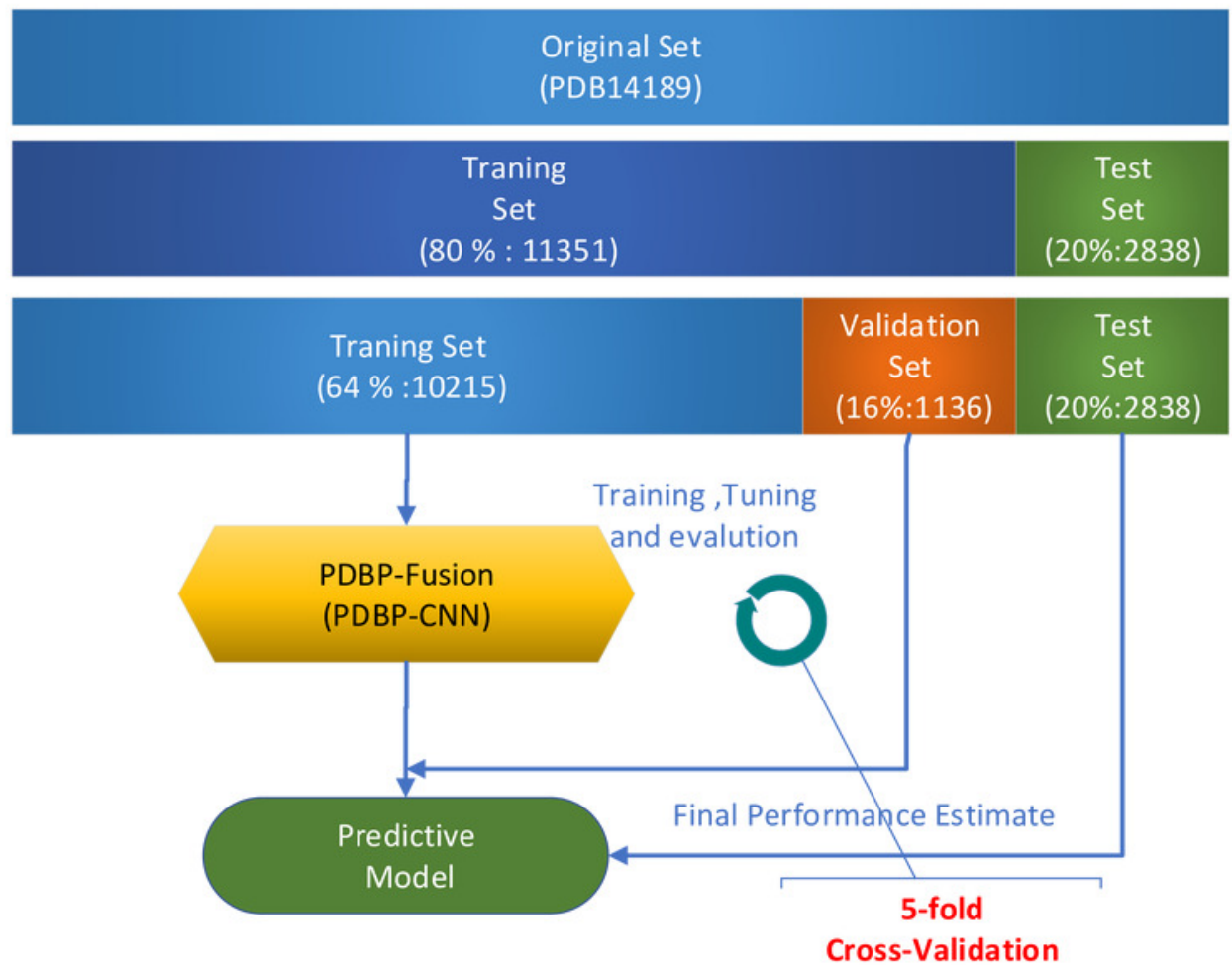


Figure 6

\section{MCCS and AUCs of the top three proposed models.}
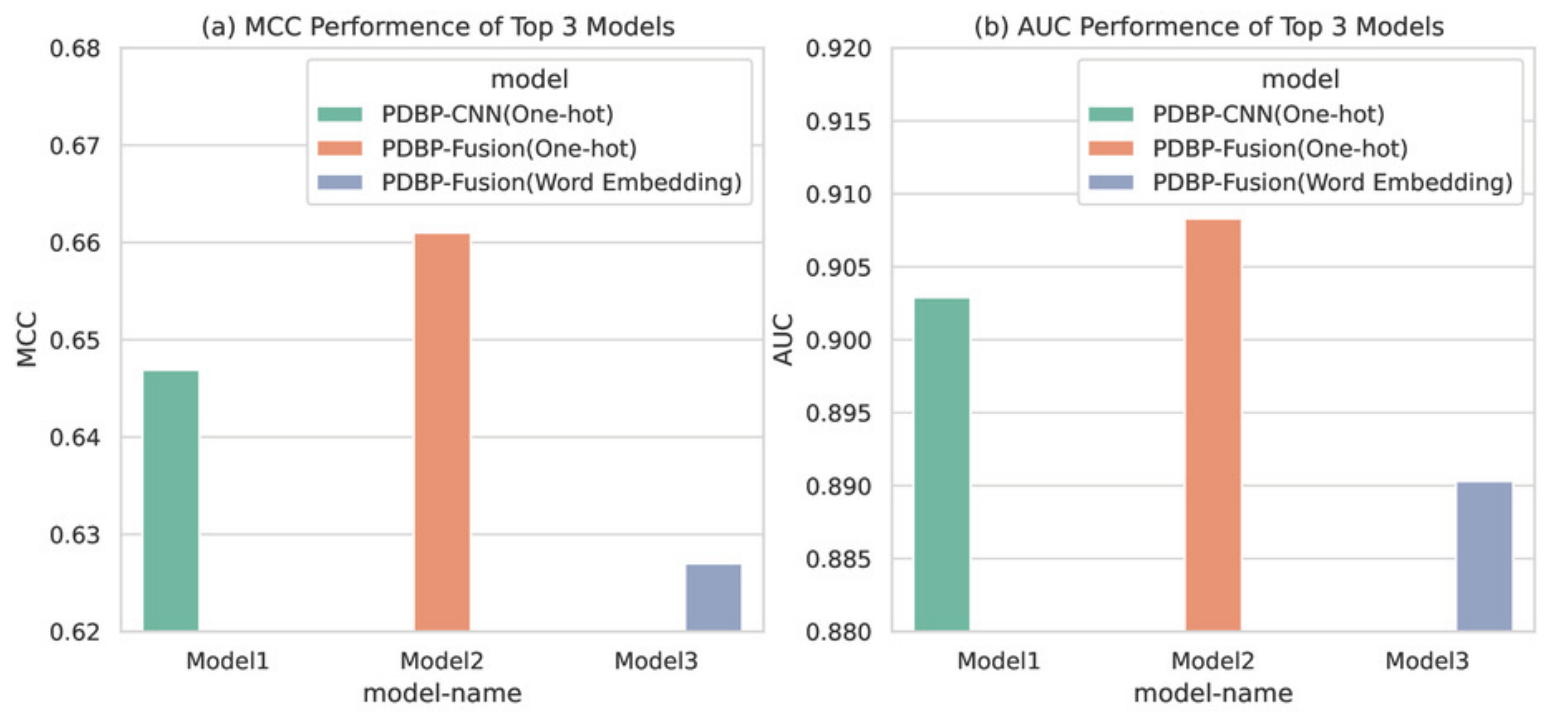


\section{Figure 7}

MCCs and AUCs of models with different dropout ratios (violin plot).

(a) MCC Performence

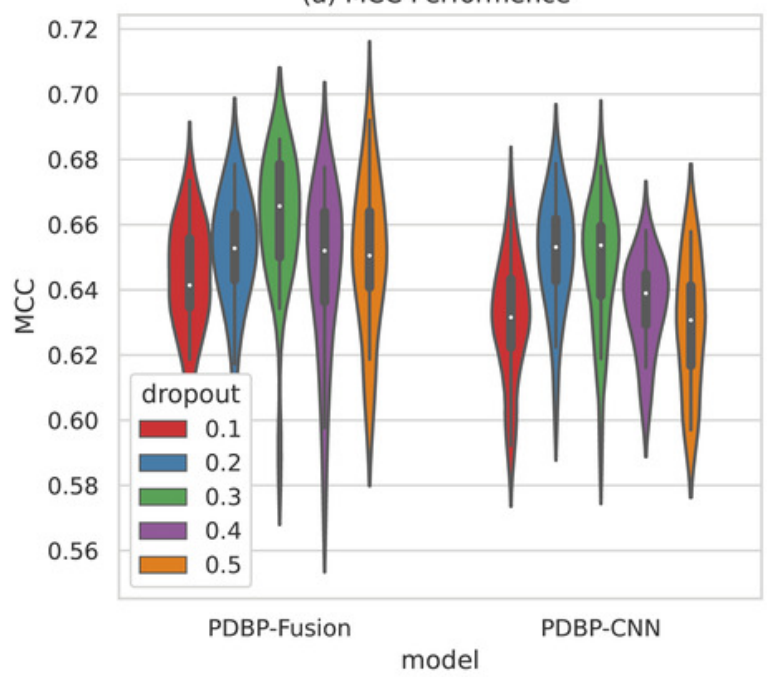

(b) AUC Performence

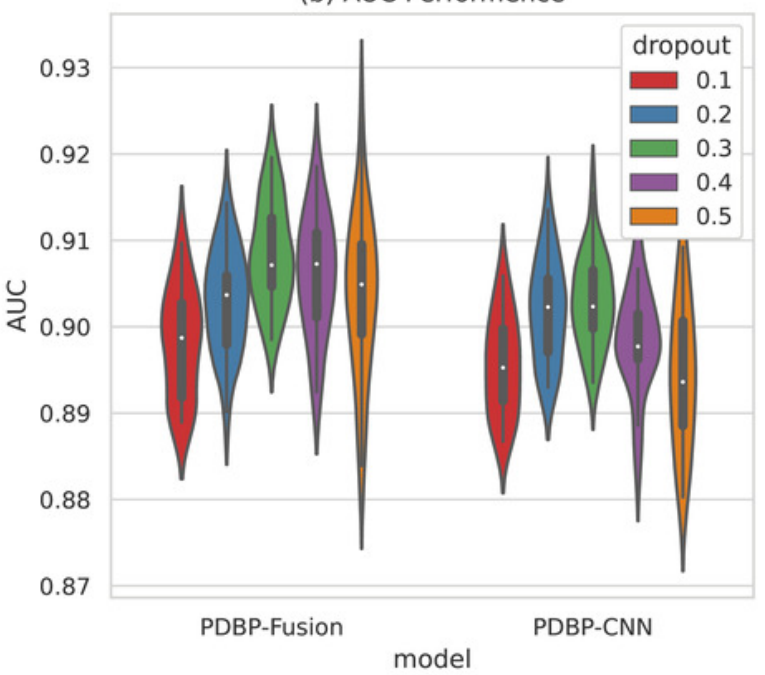


Figure 8

MCCs and AUCs of models with different dropout rates (box plot).

(a) MCC Performence

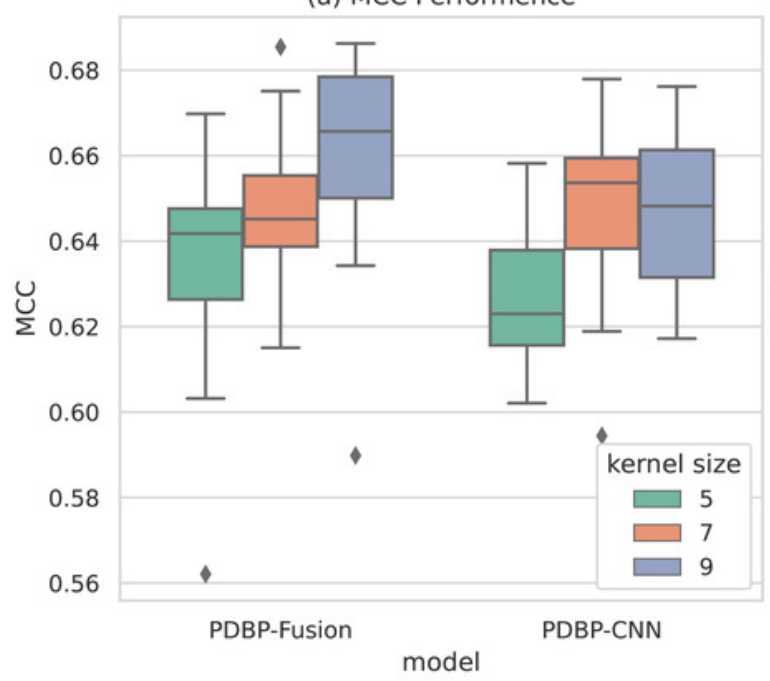

(b) AUC Performence

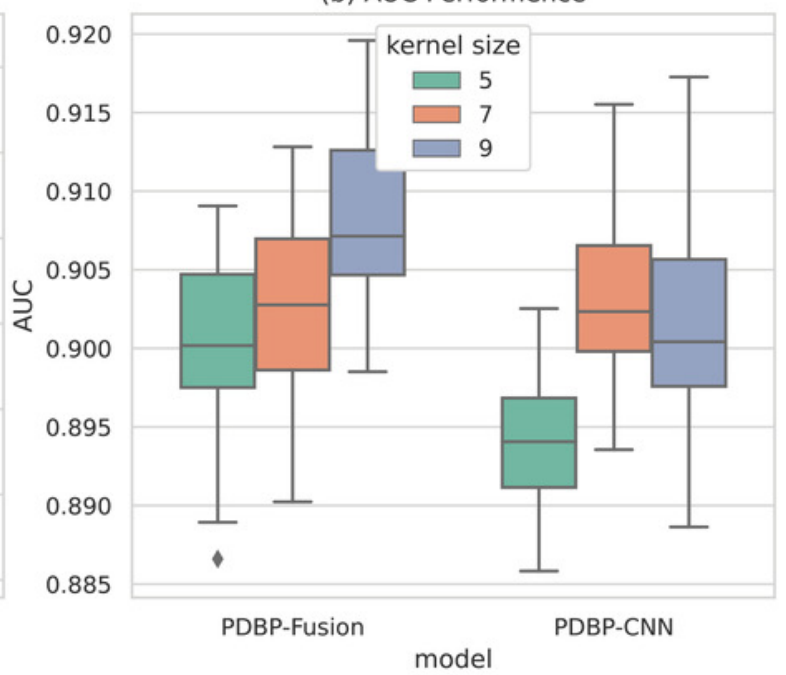


Figure 9

Overview of the StackDPPred prediction framework based on One-hot encoding. 


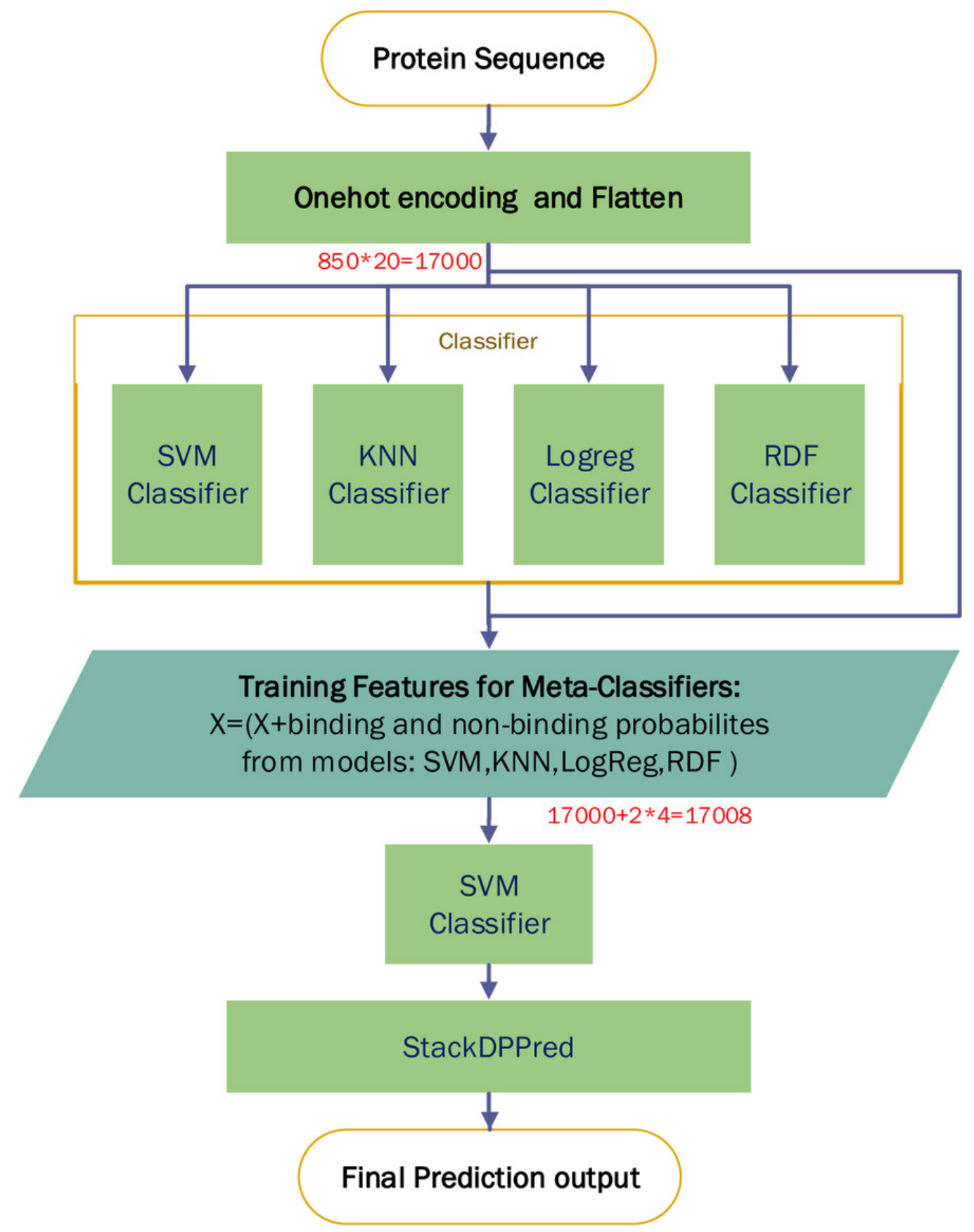




\section{Figure 10}

Index page of the Web server.

PDBP-Fusion : Prediction of DNA-Binding proteins using Local Features and Long-term Dependencies with Primary Sequence based on Deep Learning

Download

Input protein sequences:

Predict

Contact Us:any question about this, please email to dxqllp@163.com 